\section{An Unusual Presentation of Optic Disc Drusen with Optic Nerve Avulsion}

\author{
Sait Alim ${ }^{1 *}$, Selim Demir ${ }^{1}$, Erkan Gökçe ${ }^{2}$, Hüseyin Ortak', \\ Helin Deniz Demir ${ }^{1}$ and Mehmet Esen ${ }^{3}$ \\ ${ }^{1}$ Department of Ophthalmology, Gaziosmanpaşa University, Tokat, Turkey \\ ${ }^{2}$ Department of Radiology, Gaziosmanpaşa University, Tokat, Turkey \\ ${ }^{3}$ Department of Emergency, Gaziosmanpaşa University, Tokat, Turkey
}

\begin{abstract}
\section{Purpose}

To evaluate the neuroimaging and clinical findings of complete optic nerve avulsion of a patient with optic disc drusen.

\section{Methods}

A 50-year-old woman presented with left eye anterolateral luxation and vision loss after injury by a crane lifting chain. Best corrected vision acuity, slit-lamp biomicroscopy, intraocular pressure, ocular ultrasonography and neuroimaging was carried out. Results

The fundus examination revealed right eye optic disc with drusen. Vitreous hemorrhage obscured the left eye fundus examination. Computerized Tomography of the patient showed that the left eye optic disc with drusen was far from the globe when compared with the right eye.

\section{Conclusion}

The diagnosis of optic nerve avulsion could be hard in some cases. Clinicians should keep in mind that in such cases if optic disc head contains drusen the diagnosis would be easier.

Keywords: Computerized tomography; Optic disc drusen; Optic nerve avulsion
\end{abstract}

\section{Introduction}

Optic nerve avulsion is very rare condition and it is an important complication of blunt trauma to the globe. The optic nerve avulsion is most frequently secondary to motor vehicles and bicycle accidents followed by falls [1]. Sport injuries such basketball, cricket and skateboarding are rarely caused optic nerve avulsion [2]. Complete avulsion of the optic nerve mechanisms defined such as anterior

*Corresponding author: Sait Alim, Department of Ophthalmology, Gaziosmanpaşa University, Tokat, Turkey, Tel: +90 5073969257; Fax: +90 3562521625; E-mail: drsaitalim@gmail.com

Citation: Alim S, Demir S, Gökçe E, Ortak H, Demir HD, et al. (2016) An Unusual Presentation of Optic Disc Drusen with Optic Nerve Avulsion. J Ophthalmic Clin Res 3: 020.

Received: January 29, 2016; Accepted: March 30, 2016; Published: April 13, 2016 luxation of the globe, forced globe rotation or sudden rise in intraocular pressure [3].

Optic disc drusen or optic disc head drusen are found about $2.4 \%$ in a necropsy study [4]. About two thirds to three quarters of clinical cases are bilateral. Here we present a patient with bilateral optic disc with drusen who had a blunt trauma to his left eye by a crane lifting chain which caused complete optic nerve avulsion.

\section{Case Report}

A 50-year-old healthy woman presented after a crane lifting chain trauma with luxation of the left globe as well as upper eyelid lacerations (Figure 1). On local examination the left globe was displaced anterolaterally and the upper eyelid was full thickness incised. Left eye visual acuity was no light perception. Complete limitation of extraocular motility in all directions. In slit-lamp biomicroscopy, cornea was minimal edematous and anterior chamber depth was normal. There was nearly $3 \mathrm{~mm}$ hyphema in the anterior chamber. The pupil was mid-dilated and non-reactive. The Lens was intact. Vitreous hemorrhage obscured fundus examination. The right eye visual acuity was 10/10 in Snellen. Except for an absent consensual pupillary reaction and an optic disc with drusen, other findings were normal (Figure 2). Ocular Ultrasonography (US) showed an optic disc with drusen in the right eye (Figure 3). The globe was hypotonic because of the optic disc head separation from it. Therefore we did not perform US to the hypotonic eye. Left eye orbital Computerized Tomography (CT) showed proptosis, intraconal retrobulbar hemorrhage, stretching of superior extraocular muscle and optic disc with drusen. Right eye orbital CT scan showed optic disc with drusen as well. When compared with the right eye the left eye optic disc with drusen was far from the globe. This finding guided us diagnosing complete optic nerve avulsion (Figure 4). Magnetic Resonance Imaging (MRI) revealed similar findings as CT scan.

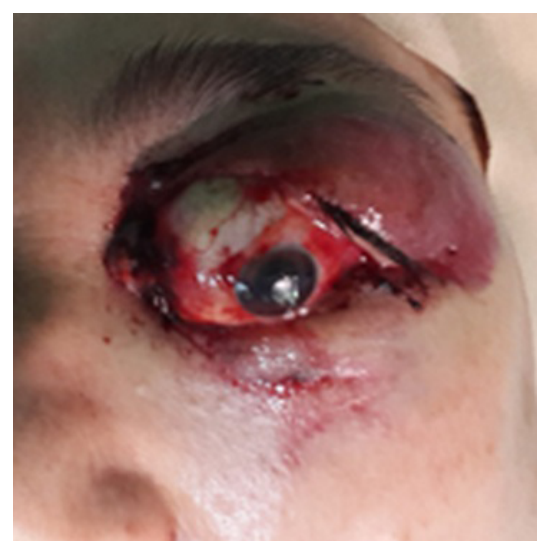

Figure 1: Anterolateral luxation of the left globe and full thickness upper eyelid lacerations.

The patient was informed and written consent was obtained. Then, the patient was brought to the operating room. The globe tried to put back in the anatomic position. The medial rectus was severed $2 \mathrm{~mm}$ far from its insertion. Medial rectus was retracted beyond the globe so we could not find the distal part of it. Therefore the proximal part 


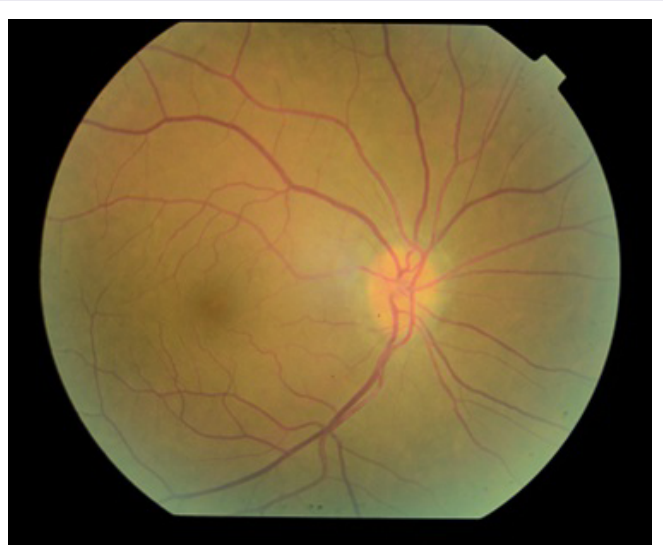

Figure 2: Fundus photograph of the right eye with optic disc drusen.

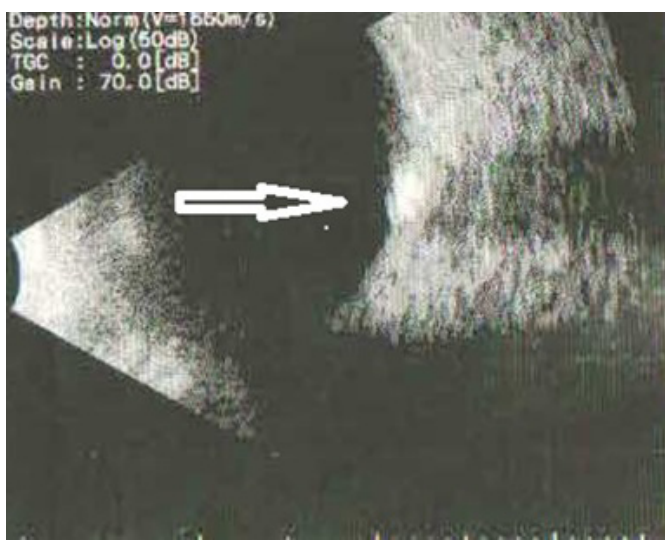

Figure 3: Ultrasonograpy of the right eye. Optic disc drusen showed by white arrow.

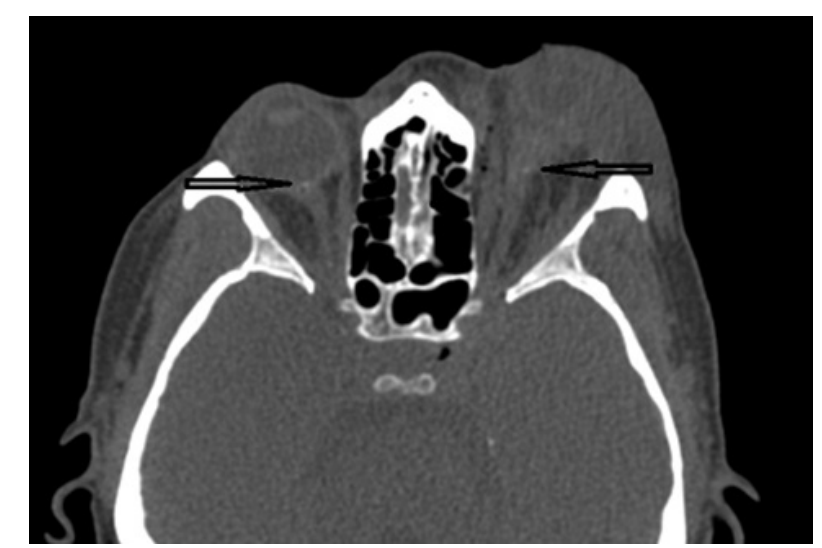

Figure 4: Computerized tomography scan of the patient. Bilateral optic disc drusen showed by black arrow. As it is seen in the CT scan the drusen is far from the left globe. Posterior border of the left globe is shown by white arrow.

of the medial rectus sutured to the bone periosteum of medial wall of the globe. The remainder of other rectus muscles insertions was intact. But the superior rectus was stretched and so it folded over itself and sutured. In the end the upper eyelid was repaired. Postoperatively the globe was in anatomic position and the patient's visual acuity was no light perception. Eye movements were restricted. The right eye examination was normal. It is a very rare case and to the best of our knowledge it is the first case evaluating optic disc head drusen with optic nerve avulsion.

\section{Discussion}

In optic nerve avulsion, the optic nerve is forcibly detached from the globe and usually there is an object that intrudes between the orbit wall and globe and dislocates the eye. It is thought that, sudden severe rotation of the globe, anterior displacement of the eyeball and rapid rise in intraocular pressure leading to expulsion of optic nerve out of scleral canal can cause optic nerve avulsion [3]. The luxation of the globe is seen rarely and following trauma medial rectus is the most frequently ruptured extraocular muscle $[5,6]$. Our patient had a trauma from medial wall of the globe and the crane lifting chain displaced the globe anteriorly. The eye was luxated and the medial rectus was severed near its insertion. If there is a clear media the diagnosis of complete optic nerve avulsion can be done easily. In such cases the disc area will be seen empty. If there is a vitreous hemorrhage or mature cataract then it would be difficult to diagnose it. In such cases it is essential to diagnosis the patient properly to avoid giving inappropriate treatment such as high-dose steroids or optic nerve decompression [3].

Optic nerve drusen is defined as hyaline bodies that result from calcification of intracellular axonal mitochondria located in the prelaminar portion of optic nerve [4]. B-scan US, a non-invasive diagnostic tool, is a reliable method for confirming the presence of optic disc drusen [7]. CT is also a useful method for diagnosis of optic disc drusen due to their calcium content.

Various diagnostic tests like MRI, CT scan, US and fundus fluorescein angiography have been used in diagnosis of optic nerve avulsion. These diagnostic tests could not always show the optic nerve avulsions. For this reason sometimes surgical exploration may needed for exact diagnosis of optic nerve avulsion. Roth et al., [8] have described a patient with optic nerve avulsion who had trauma to his eyeball by the golf club. The CT scan of brain and orbits showed no abnormalities. Foster et al., [9] carried out a study to find out the role of diagnostic imaging in patients with optic nerve avulsion. Neuroimaging including CT scan and MRI or both was done for six patients. Only in one case disinsertion of optic nerve from the globe was discovered. In two cases MRI could not reveal optic nerve avulsion. Complete optic nerve avulsion was seen just in one case when the globe was surgically explored. Therefore, it is usually hard to diagnosis optic nerve avulsion by CT scan and MRI. Our patient was also asked for orbital CT scan and MRI. Because of retrobulbar hemorrhage MRI findings was not helpful. Orbital CT scan revealed optic disc with drusen. While compared with the right eye the left eye optic disc with drusen was far from the globe (Figure 3). This finding leaded us to the diagnosis of complete optic nerve avulsion. US is also may be useful in diagnose of optic nerve avulsion. Prior studies of US have revealed encouraging results in diagnosis of optic nerve avulsion [10]. Sawhney et al., [3] had found that US of globe demonstrated hypoechoic defect in the posterior ocular coat showing complete avulsion in their two cases.

\section{Conclusion}

Optic nerve avulsion is sight threatening complication of blunt trauma. It is very important to expose optic nerve avulsion because treatment should be done according to the diagnosis. The purpose of the present study was to emphasize the importance of optic disc with drusen in diagnosis of complete optic nerve avulsion. 
Citation: Alim S, Demir S, Gökçe E, Ortak H, Demir HD, et al. (2016) An Unusual Presentation of Optic Disc Drusen with Optic Nerve Avulsion. J Ophthalmic Clin Res 3: 020.

- Page 3 of 3 -

\section{References}

1. Steinsapir KD, Goldberg RA (1994) Traumatic optic neuropathy. Surv Ophthalmol 38: 487-518.

2. Roberts SP, Schaumberg DA, Thompson $P$ (1992) Traumatic avulsion of the optic nerve. Optom Vis Sci 69: 721-727.

3. Sawhney R, Kochhar S, Gupta R, Jain R, Sood S (2003) Traumatic optic nerve avulsion: role of ultrasonography. Eye (Lond) 17: 667-670.

4. Friedman AH, Henkind P, Gartner S (1975) Drusen of the optic disc. A histopathological study. Trans Ophthalmol Soc UK 95: 4-9.

5. Ersan I, Adam M, Oltulu R, Zengin N, Okka M (2016) Traumatic luxation of the globe: A 6-year follow-up. Orbit 35: 69-71.
6. O'Toole L, Long V, Power W, O'Connor M (2004) Traumatic rupture of the lateral rectus. Eye (Lond) 18: 221-222.

7. Zaouali S, Abroug N, Khochtali S, Kahloun R, Jelliti B, et al. (2014) Optic nerve head drusen: a comparative study of $10 \mathrm{MHz}$ and $20 \mathrm{MHz}$ ultrasound probes. Int Ophthalmol 35: 229-232.

8. Roth DB, Warman R (1999) Optic nerve avulsion from a golfing injury. Am J Ophthalmol 128: 657-658.

9. Foster BS, March GA, Lucarelli MJ, Samiy N, Lessell S (1997) Optic nerve avulsion. Arch Ophthalmol 115: 623-630.

10. Talwar D, Kumar A, Verma L, Tewari HK, Khosla PK (1991) Ultrasonography in optic nerve head avulsion. Acta Ophthalmol (Copenh) 69: 121-123. 